Bulletin of Mathematical Biology (2001) 63, 885-908

doi:10.1006/bulm.2001.0249

Available online at http://www.idealibrary.com on IDE $/ \mathbf{L}^{\circledR}$

\title{
Receptor Heterogeneity and its Effect on Sensitivity and Coding Range in Olfactory Sensory Neurons
}

\author{
PETR LÁNSKÝ* \\ Institute of Physiology, \\ Academy of Sciences of the Czech Republic, \\ Vídenská 1082, \\ 14220 Prague 4, \\ Czech Republic \\ WAYNE M. GETZ \\ Division of Insect Biology, ESPM, 201 Wellman Hall, \\ University of California at Berkeley, \\ CA 94720-3112, \\ U.S.A.
}

Signal processing in the olfactory system is initiated by binding of odorant molecules to receptor molecules embedded in the membranes of sensory neurons. Most analyses of odorant-receptor interaction focus on one or more types of odorants binding with one type of receptors. Here, two basic models of this first step are investigated under the assumption that the population of receptors is not homogenous and is characterized by different activation/deactivation rates. Both, discrete and continuous variation of the rates are considered. The steady-state characteristics of the models are derived. In addition, time to crossing a threshold, defined as a response time, is also investigated. The achieved results are compared with those valid for models with the homogenous population of receptors and interpreted in terms of information coding. The obvious implications of the modeling study-that the heterogeneity of receptors enlarges the coding range and increases the sensitivity of the system-are quantified.

(C) 2001 Society for Mathematical Biology

\section{INTRODUCTION}

A fundamental, but critical question in olfaction that has not yet been answered satisfactorily is whether individual olfactory receptor neurons (ORNs) express only one or more than one type of olfactory receptor protein (ORP) in their dendritic membranes (Lancet et al., 1993b,a). Over the past decade, geneticists have become increasingly aware that a surprisingly large number of genes in animals code for

\footnotetext{
*Author to whom correspondence should be addressed. E-mail: lansky@biomed.cas.cz

$0092-8240 / 01 / 050885+24 \quad \$ 35.00 / 0$

(C) 2001 Society for Mathematical Biology
} 
ORPs (Buck and Axel, 1991; Buck, 1996; Malnic et al., 1999; see also Mombaerts, 1999): about 1000 or approximately $1 \%$ of genes in rats and mice code for ORPs, which appears to be even more diverse than genes coding for ligand receptors associated with the immune system. Recently, Duchamp-Viret et al. (1999) argued that responses of individual ORNs to several different classes of compounds (e.g., turpene, camphor, aromatic, and straight-chained ketones) imply that individual frog and rat ORNs express several ORPs. Nonpheromonal insect ORNs are also known to respond individually to a wide array of structurally different odorants [for reviews Masson and Mustaparta (1990), Smith and Getz (1994), Lemon and Getz (1999)] and it is estimated that the fruit fly genome contains on the order of 100 ORP genes [as reviewed in Mombaerts (1999)]. Malnic et al. (1999), however, present empirical evidence that in rats individual ORNs appear to express only one ORP and that it is individual ORPs themselves that are able to bind to an array of structurally diverse odorants. Here we develop a model that can help assess whether one or more subpopulations of ORPs are expressed by individual ORNs. Application of the model is not limited to ORNs, but to the transduction of chemical information across any cell membrane. Our leitmotif, however, is a generalist insect ORN located in hair-like sensilla on the antenna. These ORNs are regarded as coding olfactory information in terms of a firing rate response to both the quality and concentration of an odor stimulus (Lemon and Getz, 2000).

The process whereby an odor stimulus evokes a response in an ORN involves many steps with details varying for different kinds of ORNs (e.g., pheromonal versus general ORNs in insects), different component odorants in mixtures (e.g., some odorants lead to higher excitation than others), and differences among species of animals. Selected details of this process are known only for particular ORNs in specific species, and then only for particular steps of the process. All the critical stages involved in the response of a given class of ORNs in an identified species of animal have yet to be described.

We carry out investigations in the context of a pure odorant stimulus presented at constant levels and compare the proportion of bound ligand-receptor complexes if the ligand (odorant) in question is able to bind with ORPs that have one, several, or a distribution of different hypothetical affinities. The shape of the curve representing the proportion of bound ligand-receptor complexes as a function of ligand density (or the logarithm of ligand density) can be used as a surrogate for ORN response. The activity of ORNs can be regarded as an amplification of the weak signal resulting from the initial binding of the odorant molecules to the ORPs on the neuronal membrane. Nonlinearities in second messenger cascades and ion channel gating processes will distort the dependency of the bound complexes on the stimuli, but if this distortion is a monotonic function, which seems to be a natural presumption, of the proportions of bound ligand-receptor complexes then many of our conclusions regarding the effects of heterogeneity of ORP populations on transient and equilibrium proportions of bound ligand-receptor complexes will provide insights into the effects of ORP heterogeneity on the response of individual 
ORNs. For details on how to introduce some of these additional processes into the model, see Rospars et al. (1996) and Getz (1999).

We also have the option of selecting various levels of detail in modeling the steps leading to the odorant-receptor complexes in terms of the concentration of odorant delivered to the mucosal membrane (vertebrates) or sensilla (invertebrates) containing the afferent dendrites of the ORNs. In the case of insects (Fig. 1), for example, odorants have to traverse pores in the cuticle of the olfactory sensilla and then diffuse or to be carried by odorant binding protein (OBP) across the liquid environment of the perireceptor space to then bind with ORPs embedded in the ORN dendritic membranes. Although OBP has been found in pheromone ORNs in moths, along with other enzymes that could be involved in metabolizing 'used' odorants or restoring OBPs from their oxidized (used) state to their reduced (active) state (Kaissling, 1998). Again, the details of all the steps or even their number are not known. If OBP is involved then the following processes should to be considered (see Fig. 1):

- the transfer of odorant from the external space into the perireceptor space with rate $k_{\mathrm{I}}$ and its possible release form this space with rate $k_{-\mathrm{I}}$;

- reversible interaction between OBP (in its reduced state) and odorant in perireceptor space to form bound their complexes at rates $k_{0}$ and $k_{-0}$. An influx of OBP can be due either to restoration of of its oxidized form or to synthesis of new molecules;

- the binding of odorant-OBP complexes with ORPs on the dendritic membrane at binding and unbinding rates $k_{1}$ and $k_{-1}$ respectively.

- activation of bound receptors at rate $k_{2}$ with reversal at rate $k_{-2}$ and, in turn, the breakdown after unbinding of odorant-OPB complexes at rate $k_{3}$. We make an assumption that when activated ORP complexes break up, the odorant is in a form that is no longer accessible to OBPs.

A complete description of all known processes is too complicated for an analytical solution, especially in the context of membranes with more than one type of ORP binding to the odorant or ligand of interest. (For each type of ORP, indexed by $j, j=1, \ldots, n$, binding to odorant $i, i=1, \ldots, m$, distinct interactions governed by rates $k_{i j}$ need to be considered.) Several special cases, however, can be usefully studied. Two scenarios already thoroughly studied for homogenous populations of ORPs are extended below to the case of membranes with two or more ORP types. Each is a special case of the more general approach presented in Fig. 1, which is, of course, closer to reality but less formally tractable and transparent.

1.1. Classical concentration detector. Beidler (1962), Ennis (1991), Getz and Akers (1995), Malaka et al. (1995), Lánský and Rospars (1993, 1995), Rospars et al. (1996): in this scenario it is assumed that the neuronal membrane is directly exposed to the odorant molecules present in the external space (this is formally equivalent to letting $k_{\mathrm{I}} \rightarrow \infty$ and $k_{-\mathrm{I}} \rightarrow \infty$ ). This simplification results in a 


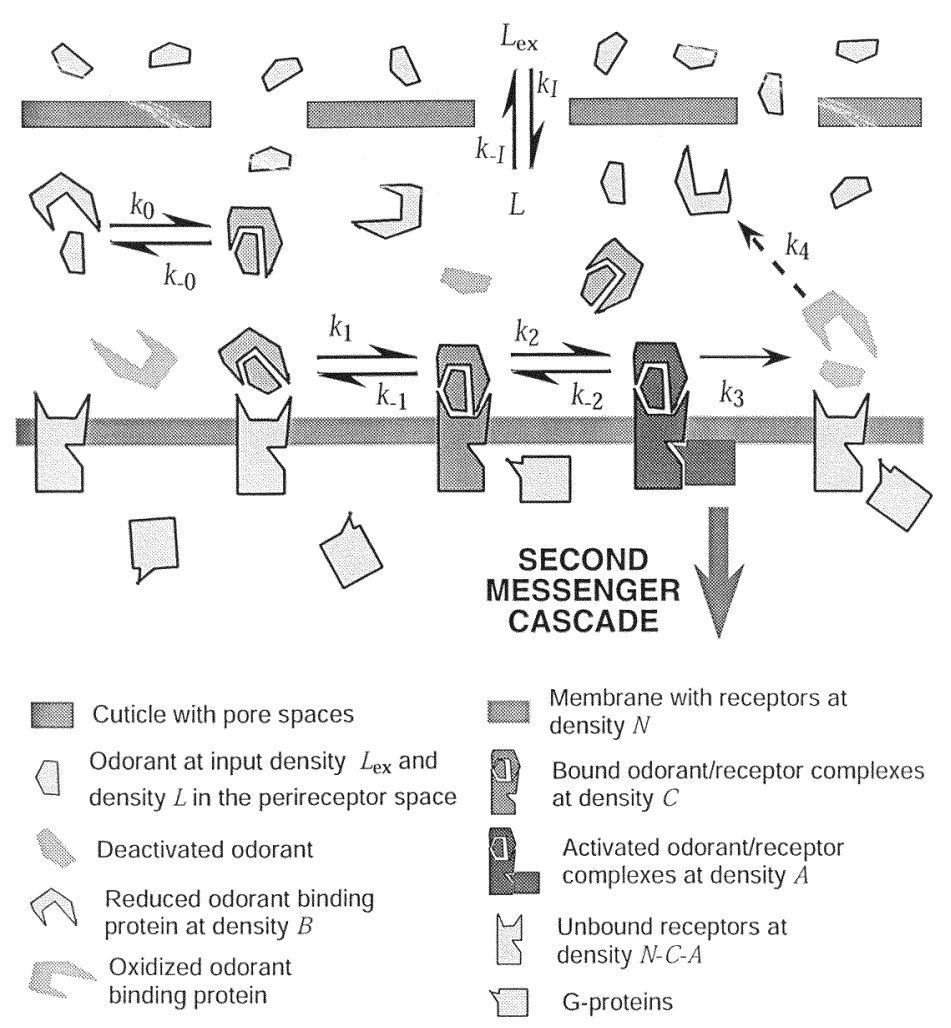

Figure 1. A cartoon of some of the critical processes involved in odorant-receptor binding in olfactory sensory neurons. For details see the text.

model which is substantially less sensitive than real neurons. By assuming very fast (relative to other reactions) and reversible transfers of odorant molecules between the external and perireceptor spaces, the model permits the actual concentration of odorant in the perireceptor space to be higher than in the external space by a factor equal to the ratio of the influx and outflux rates. More precisely, if no other steps are considered, then the ratio between the perireceptor and external concentrations is equal to $k_{\mathrm{I}} / k_{-\mathrm{I}}$ and the amplification arises if this ratio is greater than one. If this amplification is included, the sensitivity of the model can be boosted so that insensitivity is no longer a disadvantage. Further, in the classical concentration detector, the role of OBP is ignored. This is equivalent to assuming that concentration of OBP is much higher than the odorant concentration in the perireceptor space (formally equivalent to letting $k_{0} \rightarrow \infty, k_{-0}=0$ ). Also, we assume that the activation of the bound ORP is an immediate consequence of binding (formally equivalent to letting $k_{2}=k_{-2} \rightarrow \infty$ ). Finally, no degradation of the activated (bound complex) is included (i.e., we set $k_{3}=0$ ).

1.2. Flux detector. Kaissling (1998), Lánský and Rospars (1998), Rospars et al. (2000), Lánský et al. (2001): in this scenario it is assumed that the transfer of odorants between the external and perireceptor spaces is relatively slow and irre- 
versible, (this is equivalent to setting $k_{-\mathrm{I}}=0$ ). In this model the concentration of the odorant in the perireceptor space can be again substantially higher than outside. The difference between the flux and concentration detector models is based on the fact that in the flux detector model the rate of the influx is quantitatively taken into account (reflecting for example the speed of wind) and no outflux is permitted. As in the concentration detector model, we do not include OBP (formally equivalent to allowing $k_{0} \rightarrow \infty$ and $k_{-0}=0$ ). Also, the difference the between bound and activated states of ORPs is not taken into account (formally equivalent to letting $k_{2}=k_{-2} \rightarrow \infty$ ), but the degradation must be included to compensate for the fact that no possible outflow of the odorant occurs (i.e., we must have $k_{3}>0$ ).

At least two kind of problems must be solved by the ORNs: the detection problem and the intensity coding problem. Detection relates to the lowest concentration at which a response is obtained. Higher sensitivity means that lower concentrations are able to evoke a response. The intensity coding problem is related to the range of concentrations (coding range) over which graded responses are obtained. Finally, related to both of these, but mainly to the detection problem is the question of the response time. The reaction to the presence of the odorant, for obvious reasons, must not be substantially delayed after its application, even for the lowest detectable amount. Then, increasing the intensity of stimulation the response time is expected to decrease. In this article we focus on how the steady-state levels of the activated ORPs (i.e., activated ligand-ORP complexes) in both models depend on the heterogeneity of the ORP populations. This permits us to evaluate the extent to which heterogeneity of ORP populations modifies the sensitivity and odor coding range, as predicted for membranes with a homogenous population of ORPs. Additionally, we investigate the kinetics of activated ORPs in terms of the time it takes for the proportion of activated ORPs to reach a predefined level after the neuron is stimulated with a square wave input.

\section{THe Model}

Consider a patch of sensory membrane uniformly covered with $n$ types of ORPs. Let $N_{j}$ denotes the density of ORPs of type $j$, and let $R_{j}(t)$ and $C_{j}(t)$ denote the concentrations of free ('not interacting') and bound ('interacting') ORPs at time $t$, $(j=1, \ldots, n)$ i.e., $R_{j}(t)+C_{j}(t)=N_{j}$. Thus $N=\sum_{j=1}^{n} N_{j}$ is the total density of ORPs. We assume that the bound complexes are indistinguishable from one to another in terms of their contribution to membrane depolarization and thus only the total concentration

$$
C(t)=\sum_{j=1}^{n} C_{j}(t)
$$

of the bound ORPs is relevant for determining neuronal activity. As mentioned in the Introduction, here we assume that the signaling complex is created in a single 
step. Generalizations based on more than one step ( $k_{2}$ and $k_{-2}$ are both finite) follow in obvious manner despite formal complications.

2.1. The stimulus. The odorant molecules are diluted at time $t$ in the carrier medium at a concentration $L_{\mathrm{ex}}(t)$. There is an abrupt change in concentration at time $t=0$ from zero to a constant level $L_{\mathrm{ex}}>0$,

$$
L_{\mathrm{ex}}(t)= \begin{cases}L_{\mathrm{ex}} & \text { for } t \geq 0 \\ 0 & \text { for } t<0\end{cases}
$$

With this stimulation, the steady-state behavior of the system and its reaction to the stimulation onset can be investigated. The experimental results are always related to the decadic logarithm of the odorant concentration, $K_{\mathrm{ex}}=\log _{10}\left(L_{\mathrm{ex}}\right)$. Therefore, for a possible comparison, we present the results not only in terms of $L_{\mathrm{ex}}$ but in terms of $K_{\mathrm{ex}}$ as well.

2.2. Concentration detector. This is the simplest and most common model describing the early stages of information transfer in olfactory systems. The model considers no practical distinction between perireceptor and external space. As we have pointed out, a more general model is derivable by assuming that the influx and outflux rates $k_{\mathrm{I}}$ and $k_{-\mathrm{I}}$ of odorant in the perireceptor space (Fig. 1) are two or more orders of magnitude larger that other constants. Consequently, the concentration of odorant at the vicinity of the membrane, $L(t)$, is not influenced by the interaction of odorant with ORPs. From the balance equation $\frac{d L}{d t}=k_{\mathrm{I}} L_{\mathrm{ex}}-k_{-\mathrm{I}} L$ it follows that the equilibrium ratio between $L$ and $L_{\mathrm{ex}}$ is equal to $k_{\mathrm{I}} / k_{-\mathrm{I}}=\alpha$ and thus $L \gg L_{\text {ex }}$ if $k_{\mathrm{I}} \gg k_{-\mathrm{I}}$, even though $k_{-\mathrm{I}}$ itself is taken to be relatively very large. For notational simplicity we assumed that the concentration of the odorant $L$ in the perireceptor space is kept equal to the concentration $L_{\mathrm{ex}}$ in the external space, $L(t)=L_{\mathrm{ex}}(t)$, so that the amplification is $\alpha=1$.

Let us suppose that unbound ORPs $\mathrm{R}_{j}$ compete for ligands $\mathrm{L}$ through the interactions, $\mathrm{L}+\mathrm{R}_{j} \underset{k_{-1 j}}{\stackrel{k_{1 j}}{\rightleftarrows}} \mathrm{C}_{j}$, where, for $j=1, \ldots, n$, the rate constants of the binding and release, for ORP type $j$, are denoted $k_{1 j}$ and $k_{-1 j}$. Taking into account that $\sum_{j=1}^{n} R_{j}(t)+C(t)=N$ is constant, $n$ independent equations can be written for description of the system. We select the equations in $C_{j}$ as it is the quantity of interest. Thus our model is

$$
\frac{d C_{j}(t)}{d t}=-\left(k_{-1 j}+k_{1} L_{\mathrm{ex}}(t)\right) C_{j}(t)+k_{1 j} L_{\mathrm{ex}}(t) N_{j}, \quad C_{j}(0)=0 .
$$

In equation (2.3) we assume that the initial concentrations of bound ORPs are equals to zeros and it is indicated explicitly that $L_{\mathrm{ex}}$ is a function of $t$. For a step input of the form given in equation (2.2), the solution of system (2.3) is

$$
C_{j}(t)=C_{j \infty}\left(1-\exp \left(-t\left(k_{-1 j}+k_{1 j} L_{\mathrm{ex}}\right)\right)\right),
$$


where

$$
C_{j \infty}=\frac{k_{1 j} L_{\mathrm{ex}} N_{j}}{k_{-1 j}+k_{1 j} L_{\mathrm{ex}}}
$$

is the steady-state level of the activated ORPs of type $j$. The level $C_{j \infty}$ is achieved exponentially with time constant $\left(k_{-1 j}+k_{1 j} L_{\mathrm{ex}}\right)^{-1}$ and the curve $C_{j \infty}$ as a function of $L_{\mathrm{ex}}$ is hyperbolic, though as a function of $\log _{10}\left(L_{\mathrm{ex}}\right)$ its shape is sigmoid.

From equations (2.1), (2.4) and (2.5), the total density of activated ORPs is

$$
C(t)=\sum_{j=1}^{n} N \frac{k_{1 j} L_{\mathrm{ex}}}{\left(k_{-1 j}+k_{1 j} L_{\mathrm{ex}}\right)}\left(1-\exp \left(-t\left(k_{-1 j}+k_{1 j} L_{\mathrm{ex}}\right)\right)\right) p_{j},
$$

where $p_{j}=N_{j} / N$. If the number of ORPs of each type is sufficiently large so that $N_{j} / N$ reflects the probability $p_{j}$ that a randomly chosen ORP is of type $j$, then function (2.6) may be seen as the mean of the random function

$$
\Gamma(t)=N \frac{K_{1} L_{\mathrm{ex}}}{K_{-1}+K_{1} L_{\mathrm{ex}}}\left(1-\exp \left(-t\left(K_{-1}+K_{1} L_{\mathrm{ex}}\right)\right)\right),
$$

where $\operatorname{Prob}\left(K_{1}=k_{1 j} \cap K_{-1}=k_{-1 j}\right)=p_{j}$; that is, by definition, $C(t)=E[\Gamma(t)]$, where $E[$.$] is the expectation operator.$

Steady-state response. For the steady-state of the total concentration of bound ORPs, $C_{\infty}=\sum_{j=1}^{n} C_{j \infty}$, it follows from equation (2.5), that

$$
C_{\infty}=N \sum_{j=1}^{n} \frac{L_{\mathrm{ex}}}{k_{d j}+L_{\mathrm{ex}}} p_{j}
$$

where $k_{d j}=k_{-1 j} / k_{1 j}$ is called the dissociation constant. As seen from equation (2.8), the steady-state behavior of the studied model of a heterogenous system, as for the homogenous system, depends only on the ratios between dissociation and association rates. Function (2.8), as a function of $K_{\mathrm{ex}}=\log _{10}\left(L_{\mathrm{ex}}\right)$, can be written in form

$$
C_{\infty}\left(K_{\mathrm{ex}}\right)=N \sum_{j=1}^{n} \frac{10^{K_{\mathrm{ex}}}}{k_{d j}+10^{K_{\mathrm{ex}}}} p_{j} .
$$

Thus with respect to log-concentration, equation (2.8) is a sum of sigmoids centered at $k_{d j}$ with height $N_{j}$, (Fig. 2). If all consecutive steps in the signal transduction were linear, then equation (2.9) would be the theoretical counterpart to experimentally observed input-output (transfer, response) curves. However, nonlinearities in signal transduction from binding to firing of a neuron may distort the shape of the final response function.

If follows form equation(2.7) that

$$
C_{\infty}=E\left[N \frac{L_{\mathrm{ex}}}{K_{d}+L_{\mathrm{ex}}}\right]
$$




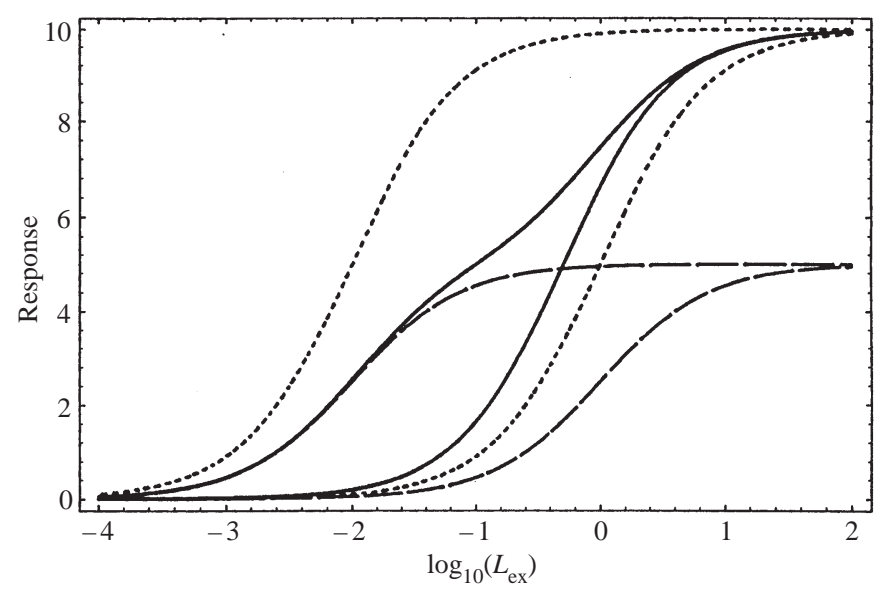

Figure 2. The concentration of activated ORPs (bound odorant-receptor complexes) referred to here as the transfer function-is plotted as a function of the decadic logarithm of stimulus intensity [i.e., $K_{\mathrm{ex}}=\log _{10}\left(L_{\mathrm{ex}}\right)$ ] for the case of homogenous and heterogenous ORP populations in the concentration detector ORN model. The heterogenous population is composed of two equally large subpopulations $N_{1}=5 \mu \mathrm{mol} 1^{-1}, N_{2}=5 \mu \mathrm{mol}^{-1}$ each respectively characterized by dissociation constants $k_{d 1}=0.01 \mu \mathrm{mol}, k_{d 2}=1 \mu \mathrm{mol}$ [solid nonsigmoid line given by (2.8) which is the sum of the two component long-dashed lines]. The two transfer functions for the two component homogenous populations regarded as a separate ORN are the two dashed lines. The transfer function for the homogenous ORN, characterized by the arithmetic mean of the dissociation constants (i.e., the parameters are $N=10 \mu \mathrm{mol} 1^{-1}$ and $k_{d}=0.505 \mu \mathrm{mol}$ ), is given by the solid sigmoid curve. Note, the transfer functions for homogenous populations of ORPs are given by equation (3.1).

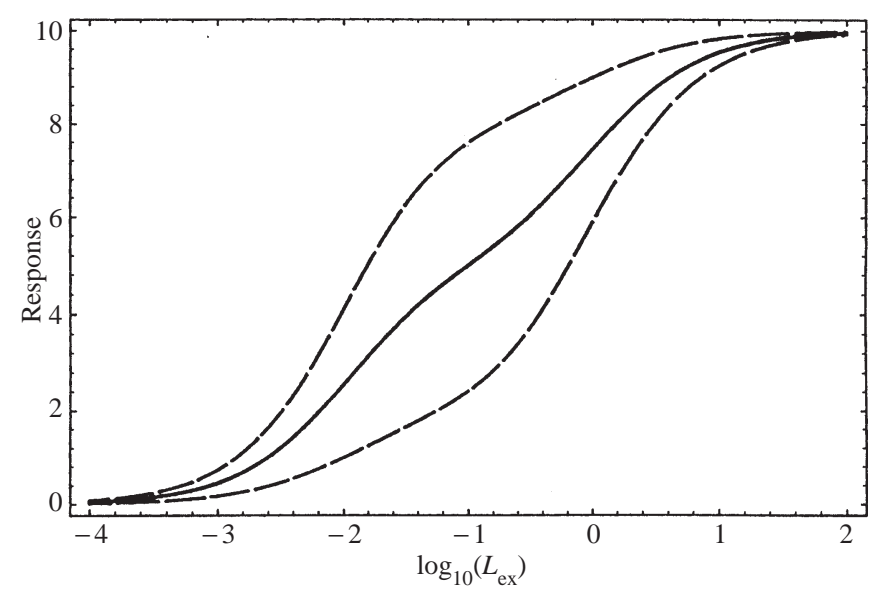

Figure 3. The mean concentration of activated ORPs (solid line) \pm two standard deviations of this random variable (dashed lines) for the concentration detector in dependency on $K_{\mathrm{ex}}$ in case of heterogenous ORP population with binomially distributed dissociation constant (parameters as in Fig. 2). 
where $K_{d}$ is a random dissociation constant. So, equations (2.8) and (2.9) can be interpreted as the response curves for a multinomial distribution of the dissociation constant, $C_{\infty}^{\mathrm{mn}}=E\left[\Gamma_{\infty}^{\mathrm{mn}}\right]$, where the superscript ' $\mathrm{mn}$ ' is used to indicate the type of distribution. The quantity $\Gamma_{\infty}^{\mathrm{mn}}$ is a random variable and thus also the moments of higher orders can be calculated. For example, the variance of $\Gamma_{\infty}^{\mathrm{mn}}$ is given in Appendix A. Note that the assumption $\operatorname{Prob}\left(K_{1}=k_{i j} \cap K_{-1}=k_{-1 j}\right)=$ $\operatorname{Prob}\left(K_{d}=k_{d j}\right)=p_{j}=N_{j} / N$ is meant to imply that ORPs having different both activation and deactivation rates cannot have the same dissociation constant (the ratio $k_{-1 j} / k_{1 j}$ ). However, this is only a notational restriction that can be easily removed.

For a cumulative distribution function $F$ of the dissociation constant $K_{d}, F(x)=$ $\operatorname{Prob}\left(K_{d} \leq x\right)$, the input-output curve defined by equation (2.10) can be written in the form

$$
C_{\infty}^{\mathrm{x}}=N \int_{0}^{\infty} \frac{L_{\mathrm{ex}}}{x+L_{\mathrm{ex}}} d F(x)
$$

where the superscript ' $\mathrm{x}$ ' is used to denote the generic form of the probability distribution (e.g., for multinomial, $\mathrm{x}$ is replaced by $\mathrm{mn}$, for uniform $-\mathrm{u}$, for normal-n).

If the number of ORP type is large, a continuity approximation would lead us to the assumption that the population of ORPs is not divided into several subgroups with substantially different parameters, but that the heterogeneity is reflected by continuous fluctuations of the dissociation constant. The same condition can be deduced from assuming that the population of ORPs is homogenous, but susceptible to random fluctuations. These fluctuations can be both small or large and this will be reflected by dispersion of the dissociation constant, $\operatorname{Var}\left(K_{d}\right)$ (i.e., variance of the dissociation constant). Continuous fluctuation of the dissociation constant induces existence of the probability density function $f(x)=d F(x) / d x$. Examples of steady state responses for uniform and normal densities are presented in Appendix A and later illustrated in Fig. 4.

Let us investigate the mutual positions of the steady-state transfer curves for homogenous and heterogenous populations of ORPs. For symmetric binomial distribution of $K_{d},\left[\operatorname{Prob}\left(K_{d}=k_{1}\right)=\operatorname{Prob}\left(K_{d}=k_{2}\right)=\frac{1}{2}\right]$ we have (see Fig. 2)

$$
\frac{1}{2} \sum_{j=1}^{2} \frac{L_{\mathrm{ex}}}{k_{j}+L_{\mathrm{ex}}}>\frac{L_{\mathrm{ex}}}{\frac{1}{2} \sum_{j=1}^{2} k_{j}+L_{\mathrm{ex}}} .
$$

In general, for any random $K_{d}$ with symmetric or asymmetric cumulative probability distribution function $F$ defined on $[0, \infty)$, for which $E\left[K_{d}\right]=k_{d}$, it can be shown (see Appendix B) that

$$
C_{\infty}^{\mathrm{x}}>N \frac{L_{\mathrm{ex}}}{k_{d}+L_{\mathrm{ex}}} .
$$

This fact will be related later to the sensitivity of the system, (see Figs 2 and 4). Inequality (2.13) implies that the number of activated ORPs in heterogenous population is always higher than the number of activated ORPs in homogenous population 


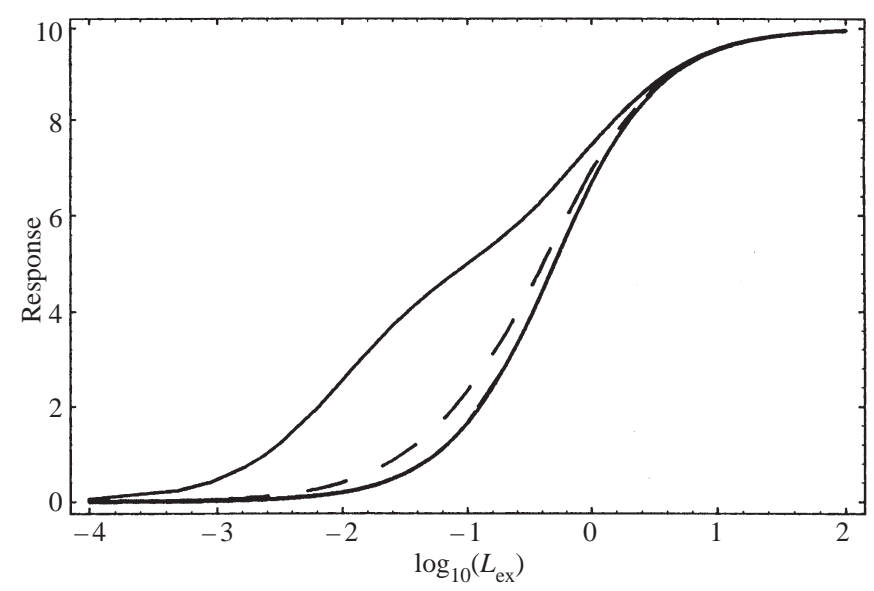

Figure 4. The mean concentration of activated ORPs in the concentration detector for uniform distribution of dissociation constant with mean $0.505 \mu \mathrm{mol}$ and $\epsilon=0.4950 \mu \mathrm{mol}$ calculated as a function of $L_{\mathrm{ex}}$ using expression (A2) (dashed line), is plotted in terms of $K_{\mathrm{ex}}=\log _{10}\left(L_{\mathrm{ex}}\right)$. For comparison, the mean concentration of activated ORPs is plotted for the binomial distribution of dissociation constant, as in Fig. 2. (solid nonsigmoid curve), and for a homogenous population with $k_{d}=0.505 \mu \mathrm{mol}$ (solid sigmoid line). The case of heterogenous population with $\epsilon=0.05 \mu \mathrm{mol}$ is not distinguishable from the homogenous population.

with dissociation constant equal to the mean dissociation constant of the heterogenous population. Visualizing this result leads to realization that the 'mean' curve in the case of two equal subpopulations of ORPs is not centered between the curves of the corresponding two homogenous cases (Fig. 2). On a logarithmic scale, a more symmetrical picture arises because the expressions $10^{K_{\mathrm{ex}}} /\left(k_{d j}+10^{K_{\mathrm{ex}}}\right)$ are symmetric functions of $K=K_{\text {ex }}$ with respect to a curve of slope -1 through their center $K_{\mathrm{ex}}=\log _{10} k_{d j}$, while the expressions $L_{\mathrm{ex}} /\left(k_{d j}+L_{\mathrm{ex}}\right)$ are not symmetric functions of $L=L_{\text {ex }}$ [cf. equations (2.8) and (2.9)]. Thus, for purposes of comparison, we derive the results in terms of a distribution for $\log _{10} K_{d}$. Intuitively we might require that $E\left(\log _{10} K_{d}\right)=\log _{10} k_{d}$. This, however, would imply that $E\left(K_{d}\right)>k_{d}$. For example, if Prob $\left(\log _{10} K_{d}=0\right)-\operatorname{Prob}\left(\log _{10} K_{d}=-2\right)=1 / 2$, then $E\left(K_{d}\right)=0.505$, but $E\left(\log _{10} K_{d}\right)=-1$, which implies $k_{d}=0.1$. For symmetric binomial distribution of $K_{d}, \operatorname{Prob}\left(\log _{10} K_{d}=a\right)=\operatorname{Prob}\left(\log _{10} K_{d}=b\right)=$ $1 / 2$, it can be shown that (see Fig. 5)

$$
\frac{1}{2}\left(\frac{10^{K_{\text {ex }}}}{10^{a}+10^{K_{\text {ex }}}}+\frac{10^{K_{\text {ex }}}}{10^{b}+10^{K_{\text {ex }}}}\right)>\frac{10^{K_{\text {ex }}}}{10^{(a+b) / 2}+10^{K_{\text {ex }}}}
$$

whenever $K_{\mathrm{ex}}<(a+b) / 2$ and reverse inequality holds whenever $K_{\mathrm{ex}}>(a+b) / 2$.

If we now generalize by assuming that $\log _{10} K_{d}$ is uniformly distributed on $\left(\log _{10} k_{d}-\epsilon, \log _{10} k_{d}+\epsilon\right)$, then the probability density function for $K_{d}$ is

$$
f(x)=\frac{1}{2 \epsilon x \ln 10}, \quad x \in\left(10^{\log _{10} k_{d}-\epsilon}, 10^{\log _{10} k_{d}+\epsilon}\right) .
$$



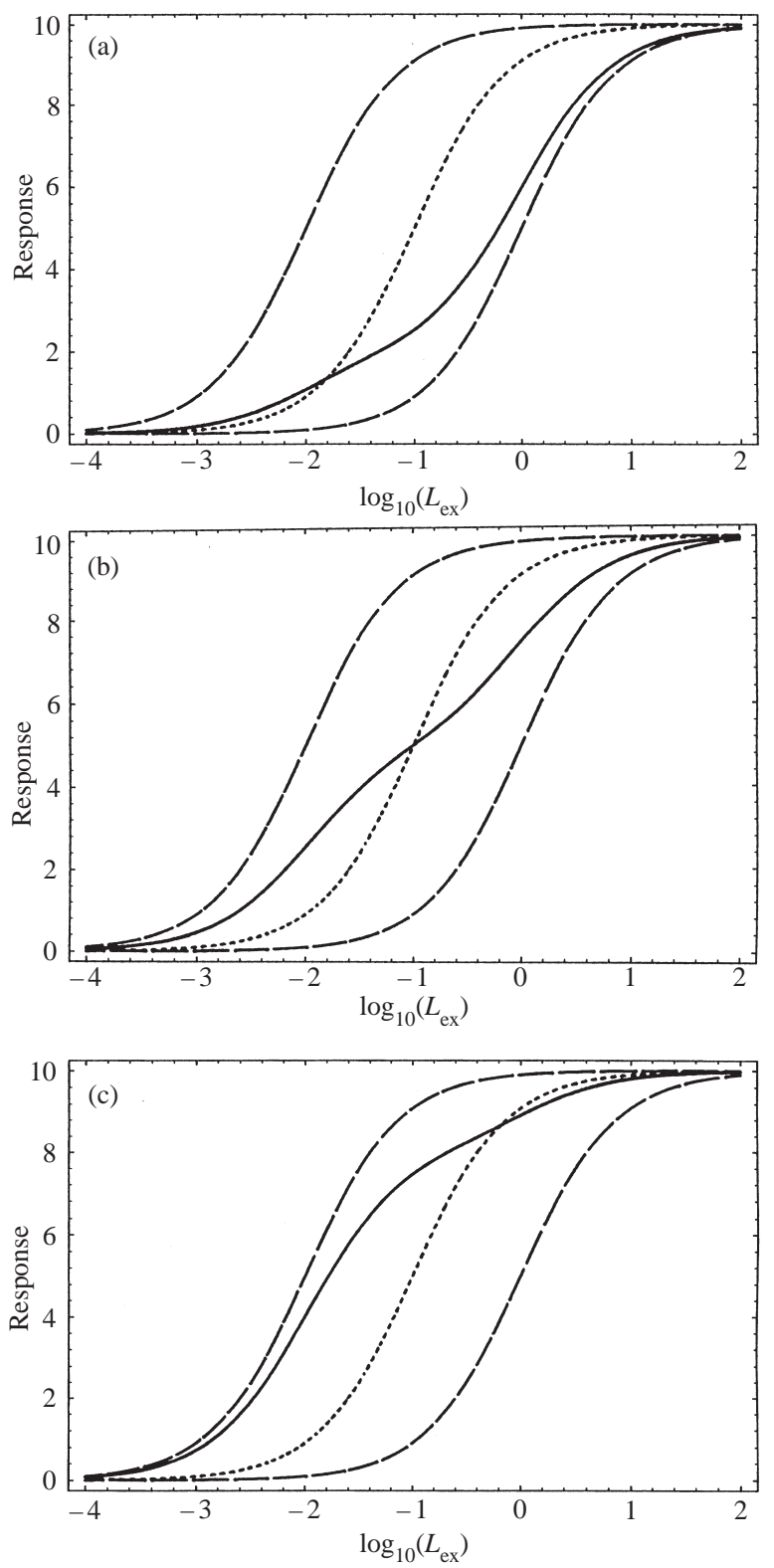

Figure 5. The mean concentration of activated ORPs in the concentration detector is plotted as a function of $K_{\mathrm{ex}}=\log _{10}\left(L_{\mathrm{ex}}\right)$ for ORNs with both homogenous and heterogenous populations of ORPs. The heterogenous ORPs are composed of two distinct subpopulations of ORPs with concentrations $N_{1}$ and $N_{2}$, and corresponding dissociation constants $k_{d 1}=0.01 \mu \mathrm{mol}, k_{d 2}=1 \mu \mathrm{mol}$. The homogenous populations are characterized by the parameters $N=10 \mu \mathrm{mol} 1^{-1}$, and in one case $k_{d 1}=0.01 \mu \mathrm{mol}$ [most left long-dashed line in (a), (b) and (c)] and in the other case $k_{d 2}=1 \mu \mathrm{mol}$ [most right long-dashed line in (a), (b) and (c)] and $k_{d}$ being the geometric mean of the dissociation constants $k_{d 1}$ and $k_{d 2}$, $k_{d}=0.1 \mu \mathrm{mol}$ [center short-dashed line in (a), (b) and (c)]. The plots for the heterogenous ORPs are: (a) $N_{1}=2 \mu \mathrm{mol} 1^{-1}, N_{2}=8 \mu \mathrm{mol}^{-1}$, (b) two equally large subpopulations $N_{1}=5 \mu \mathrm{mol} 1^{-1}, N_{2}=5 \mu \mathrm{mol} 1^{-1}$ and (c) $N_{1}=8 \mu \mathrm{mol} \mathrm{l}^{-1}, N_{2}=2 \mu \mathrm{mol} \mathrm{l}^{-1}$. 


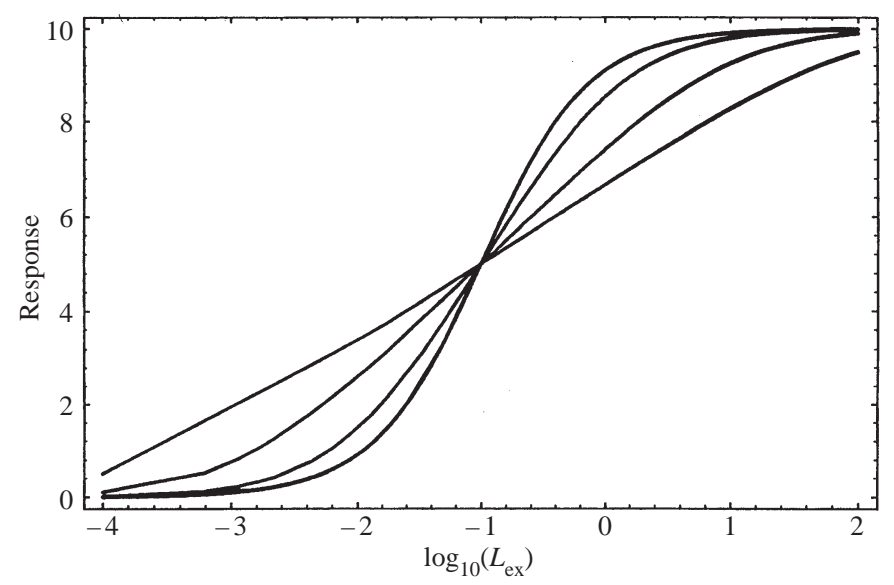

Figure 6. The mean concentration of activated ORPs in the concentration detector is plotted as a function of $K_{\mathrm{ex}}$ for the uniform distribution of decadic logarithm of the dissociation constant with mean $E\left(\log _{10} K_{d}\right)=-1$ and $\epsilon=3$ (straight line), $\epsilon=2, \epsilon=1, \epsilon=$ 0.01 (coincides with transfer function of homogenous population of ORPs), calculated from (2.16). By decreasing $\epsilon$ the transfer function approaches the sigmoid function. The rest of the parameters as in Fig. 2.

Thus, by substituting $f(x)$ into equation (2.11), we obtain

$$
C_{\infty}^{\ln \mathrm{u}}=N\left(1-\frac{1}{2 \epsilon} \log _{10}\left(\frac{k_{d} 10^{\epsilon}+L_{\mathrm{ex}}}{k_{d} 10^{-\epsilon}+L_{\mathrm{ex}}}\right)\right),
$$

which tends to $L_{\mathrm{ex}} /\left(k_{d}+L_{\mathrm{ex}}\right)$ as $\epsilon \rightarrow 0$. Again, the relative positions of the curves is analogous to that in equation (2.14) (Fig. 6).

In general, for homogenous and heterogenous systems it is possible to derive a relationship similar to (2.13), assuming that the distribution of $\log _{10} K_{d}$ is symmetric. For the cumulative distribution function $F(x)=\operatorname{Prob}\left(\log _{10} K_{d} \leq x\right)$, a symetricity assumption implies that $F(\mu-x)=1-F(\mu+x)$, [if density exists, then $f(\mu-x)=f(\mu+x)$ ], where $\mu=E\left(\log _{10} K_{d}\right)$. In this case, it holds that

$$
C_{\infty}^{x}\left(L_{\mathrm{ex}}\right)\left\{\begin{array}{lll}
>N \frac{L_{\mathrm{ex}}}{\mu+L_{\mathrm{ex}}} & \text { for } & L_{\mathrm{ex}}<\mu, \\
=\frac{N}{2} & \text { for } & L_{\mathrm{ex}}=\mu, \\
<N \frac{L_{\mathrm{ex}}}{\mu+L_{\mathrm{ex}}} & \text { for } & L_{\mathrm{ex}}>\mu .
\end{array}\right.
$$

Relationship (2.17) is illustrated in Fig. 6, where uniform distribution of $\log _{10} K_{d}$ with different spreads are used. The symmetry of $f$ implies that the distribution of $K_{d}$ is positively skewed, which holds true for all the most common distributions defined on $[0, \infty)$. Further, it induces symmetry in $C_{\infty}^{\mathrm{x}}\left(K_{\mathrm{ex}}\right)$ around the concentration at which half of the ORPs are activated.

The assumption that $K_{d}$ is a random variable following some common distribution (e.g., multinomial, normal, or uniform) could also imply that only the unbinding rate $K_{-1}$, or only the binding rate $K_{1}$ is random, rather than the ratio $K_{d}$ itself. For example see Appendix C and Fig. 7. 


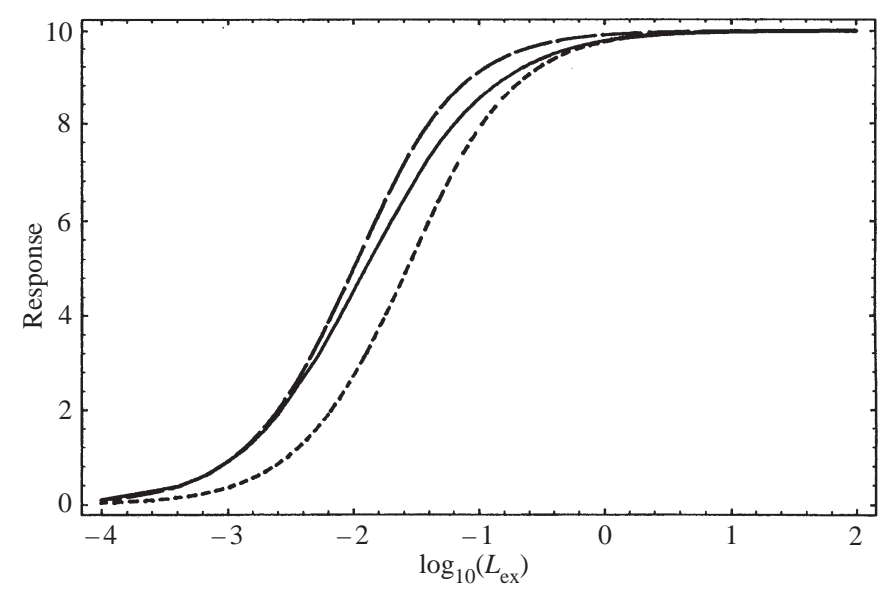

Figure 7. The mean concentration of activated ORPs in the concentration detector is plotted as a function of $K_{\mathrm{ex}}$ for heterogenous population of ORPs with uniform distribution of decadic logarithm of association rate with mean $1 \mu \mathrm{mol}^{-1} \mathrm{~s}^{-1}$ and dissociation rate $0.01 \mathrm{~s}^{-1}, \epsilon=0.99 \mu \mathrm{mol}^{-1} \mathrm{~s}^{-1}$, calculated from expression (2.5) (full line). For comparison, we plot concentration of activated ORPs for a homogenous population with $k_{d}=0.01$ (dashed line) and for the case when $k_{d}$ is the mean of distribution (C1) (dotted line), is presented.

Response time. We now turn our attention to the question of the length of time, $\delta\left(L_{\mathrm{ex}}\right)$, that it takes as a function of concentration $L_{\mathrm{ex}}$ for the proportion of activated ORPs to reach a critical threshold $S$ needed to trigger an 'on' response downstream. For a homogenous population of ORPs $(n=1) \delta$ is obtained by solving equation $C(\delta)=S$ following from equation (2.4) for $j=1$ to obtain

$$
\delta=-\frac{1}{k_{-1}+k_{1} L_{\mathrm{ex}}} \ln \left(1-\frac{S\left(k_{-1}+k_{1} L_{\mathrm{ex}}\right)}{k_{1} L_{\mathrm{ex}} N}\right) \approx \frac{S}{k_{1} L_{\mathrm{ex}} N} .
$$

The approximation holds reasonably well for the thresholds far below the asymptotic level of the concentration of the signaling complexes, $S \ll N L_{\mathrm{ex}} /\left(k_{d}+L_{\mathrm{ex}}\right)$. In other words, for sufficiently strong stimulations the response time does not depend on the dissociation rate. On the other hand, times to reach levels close to the steady-state depend strongly on the dissociation rate.

For populations composed of different ORPs, an analytical solution analogous to formula (2.18) is not available and only approximations can be used. The approximation valid in this case is obtained from the equation $C(\delta)=\sum_{j=1}^{n} C_{j}(\delta)=S$ and, as in deriving equation (2.18), it is

$$
\delta \approx \frac{S}{L_{\mathrm{ex}} \sum_{j=1}^{n} k_{1 j} N_{j}} .
$$

Using the notation $p_{j}=N_{j} / N$, equation (2.19) can be written in the form

$$
\delta \approx \frac{S}{L_{\mathrm{ex}} N E\left(K_{1}\right)},
$$


where $K_{1}$ has a multinomial distribution. The primary conclusion from this analysis is that if the heterogeneity is characterized by different dissociation rates then, as in the homogenous ORP population case, for sufficiently strong stimulation only the means of the association rates determine the response time. From convexity of function (2.20) it follows that the reaction time of ORN with heterogenous population of ORPs is shorter than that for ORN with the homogenous population in which the association rate is equal to the mean of the association rates characterizing the subpopulations, [Fig. 8(b)]. This suggests that the improvement of the response time with respect to the homogenous populations of ORPs exists.
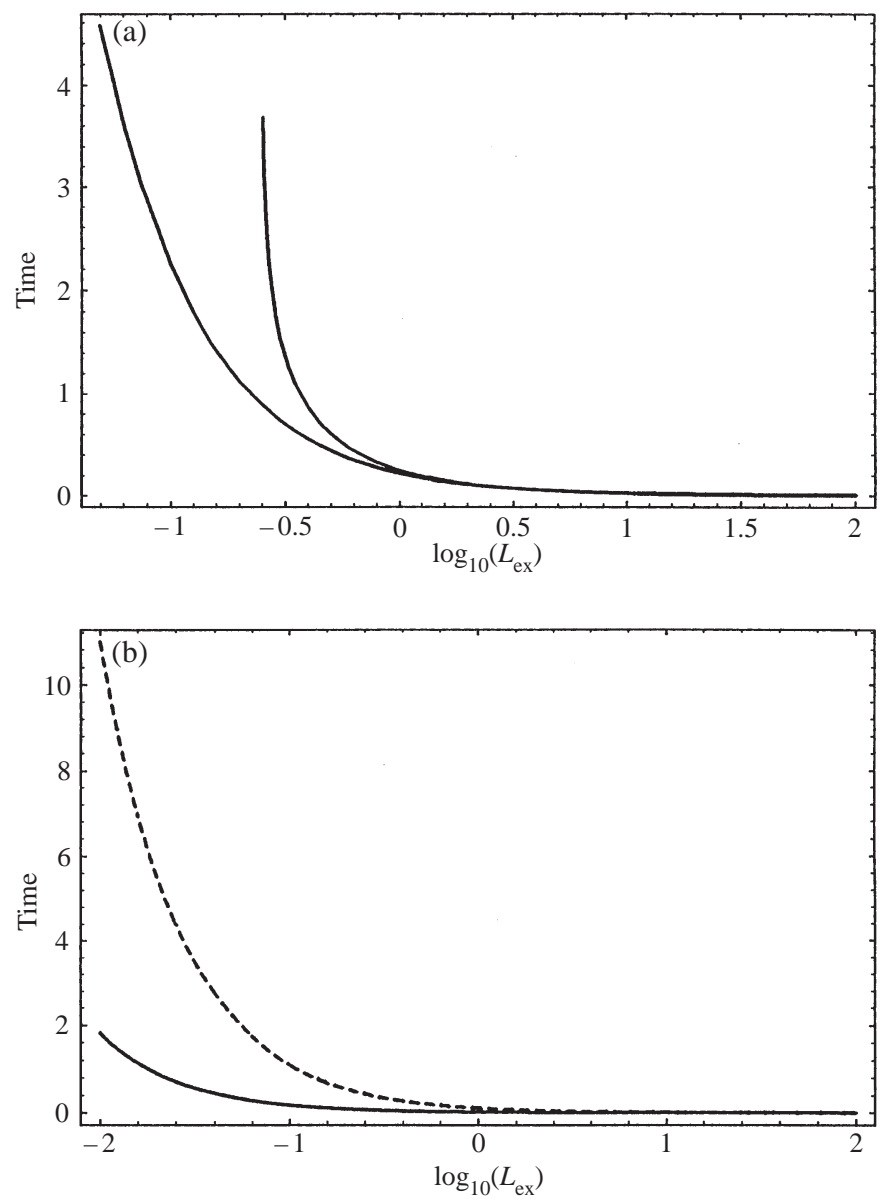

Figure 8. The reaction times $\delta$ are plotted as functions of $K_{\mathrm{ex}}$ : (a) two homogenous populations - the components of the heterogenous population of ORPs with parameters given in Fig. 2. The reaction times are given by expression (2.18). (b) Approximation of the reaction time given in expression (2.19) for a homogenous population (dashed line) and expression (2.18) for a heterogenous population (full line) characterized by the same mean association rate as the homogenous population. The parameters are $N_{1}=5 \mu \mathrm{moll}^{-1}$, $N_{2}=5 \mu \mathrm{mol} \mathrm{l}^{-1}, k_{11}=10 \mu \mathrm{mol}^{-1} \mathrm{~s}^{-1}, k_{12}=1 \mu \mathrm{mol}^{-1} \mathrm{~s}^{-1}, N=10 \mu \mathrm{mol} \mathrm{l}^{-1}$, $k_{1}=5.5 \mu \mathrm{mol}^{-1} \mathrm{l}^{-1}, S=15 \mu \mathrm{mol} 1^{-1}$. 
2.3. Flux detector. In contrast with the concentration detector, where there was no distinction between the external space and perireceptor space, it is now assumed that the vicinity of the membrane is physically distinguishable from the external space (see Fig. 1). Further, in accordance with Kaissling (1998), it is assumed that this transfer is unidirectional-from outside to inside. Two direct consequences of these assumptions are the dependency of $L(t)$ on the interaction of $\mathrm{L}$ with $\mathrm{R}$ and the necessity of degrading the ligand molecules, to prevent their unbounded accumulation in the perireceptor space. The main effect of the perireceptor space existence is in shifting the model transfer function to the left (higher sensitivity) along the stimulus concentration axis as many decades as required. Physically, this is enabled by accumulation of odorant molecules at the perireceptor space at much higher concentration than at the external space. Of course, this step must be very fast not to delay the response time. On the other hand, we should keep in mind that this approach is oriented mainly on odorant detection. Both transfer functions (flux and concentration detectors) have a similar shapes in the left part of their coding ranges, for higher concentration the flux detector fails to describe the system behavior, for detailed comparison of the models see Rospars et al. (2000).

The reaction schema describing the flux detector with heterogenous population of ORPs is $\mathrm{L}_{\mathrm{ex}} \stackrel{k_{\mathrm{I}}}{\rightarrow} \mathrm{L}, \mathrm{L}+\mathrm{R}_{j} \underset{k_{-1 j}}{\stackrel{k_{1 j}}{\rightleftarrows}} \mathrm{C}_{j} \stackrel{k_{\mathrm{oj}}}{\rightarrow} \mathrm{R}_{j}+\bar{L}$, where $\bar{L}$ represents a degraded form of $\mathrm{L}$ that cannot interact with $\mathrm{R}, k_{1}$ and $k_{\mathrm{o} j}$ are the rates of the influx and degradation. The influx to the system is $k_{\mathrm{I}} L_{\mathrm{ex}}(t)$. Let us first assume that the degradation process is independent of the ORP type, $k_{\mathrm{o} j}=k_{\mathrm{o}}$ and thus the outflow is $k_{\mathrm{o}} C(t)$. Using the same notation as before and taking into account, that $R_{j}(t)+$ $C_{j}(t)=N_{j}$ is constant, only two independent equations can be written for each fixed $j$, e.g.,

$$
\begin{aligned}
\frac{d C_{j}(t)}{d t} & =-\left(k_{-1 j}+k_{\mathrm{o}}+k_{1 j} L(t)\right) C_{j}(t)+k_{1 j} L(t) N_{j}, \\
C_{j}(0) & =0 \\
\frac{d L(t)}{d t} & =k_{\mathrm{I}} L_{\mathrm{ex}}(t)-\sum_{j=1}^{n}\left(k_{1 j} L(t) N_{j}-\left(k_{1 j} L(t)+k_{-1 j}\right) C_{j}(t)\right), \\
L(0) & =0 .
\end{aligned}
$$

With step stimulus (2.2), the system can reach a stationary state only if condition

$$
k_{\mathrm{I}} L_{\mathrm{ex}}<k_{\mathrm{o}} N
$$

holds, which means that the influx to the perireceptor space must be smaller than the maximum possible flow out of this space. Under the condition (2.23), the steady-state solution of equations (2.21) and (2.23) is

$$
C_{\infty}=\frac{k_{\mathrm{I}} L_{\mathrm{ex}}}{k_{\mathrm{o}}} .
$$


We can see that the diversity of binding and unbinding rates play no role in the steady-state behavior of the flux detector. This, seemingly paradoxical result, is due to the fact that the odorant concentration in the perireceptor space $L$ can increase above the odorant concentration $L_{\mathrm{ex}}$ and, for example, a low binding rates is finally compensated by high perireceptor space concentration of the odorant. Of course, equation (2.24) describes only the steady state behavior of the system and the situation is entirely different when the dynamical properties are investigated.

For $n=1$ and at low concentrations, equation (2.5) can be written as $C_{\infty} \approx$ $\left(N k_{1} L_{\mathrm{ex}}\right) / k_{-1}$. Thus we can see exactly the same qualitative behavior of both models at low concentrations. However, the meaning of the term 'low concentration' is different and determined by the ratio $\left(N k_{1}\right) / k_{-1}$ for the concentration detector and by the ratio $k_{\mathrm{I}} / k_{\mathrm{o}}$ for the flux detector. Over the whole range of concentrations the models behave differently. The flux detector does not result in the hyperbolic-like (sigmoid in log scale) dependency of the response on the external concentration and this result is true irrespectively to the diversity of ORPs because the shape of the curve is independent of the rates $k_{-1}$ and $k_{1}$.

\section{Coding Properties}

In the previous section, several analytical results for two basic models of the first stage in olfactory signal transduction were derived. Heterogenous populations of ORPs were assumed in both models. The investigation was performed without any specific reference to the implications for coding, which we now consider.

We have shown that the flux detector, despite its importance in making the model more realistic and suitable for identification of biophysical parameters using empirical data, does not produce qualitatively different behavior from the concentration detector. Thus we restrict our attention to the analysis of the concentration detector. From the concentration detector assumption it follows that ranges $L_{\mathrm{ex}}$ to which the models can be exposed are different. Therefore, if the following results are interpreted in the flux detector context, the range of applied concentration would be substantially lower (Rospars et al., 2000). For purpose of illustration, the following constants were used, except where otherwise specified: $10^{-4} \leq L_{\mathrm{ex}} \leq$ $10^{2} \mu \mathrm{mol} \mathrm{l}^{-1}$ for the range of odorant concentration, $N=10 \mu \mathrm{mol} \mathrm{l}^{-1}$ for the concentration of ORPs, and $k_{1}=1 \mu \mathrm{mol}^{-1} \mathrm{~s}^{-1}$ and $k_{-1}=1 \mathrm{~s}^{-1}\left(k_{-1}=0.01 \mathrm{~s}^{-1}\right)$ respectively for the activation and deactivation rates.

The traditional understanding of sensitivity is based on identifying the concentration of odorant at which the sigmoid curve for homogenous ORP case [i.e., $n=1$, cf. equation (2.9)—also recall $K_{\text {ex }}=\log _{10}\left(L_{\text {ex }}\right)$ and note that $\delta\left(k_{d}\right)$ represents a Dirac function positioned at $k_{d}$ ]

$$
C_{\infty}^{\delta\left(k_{d}\right)}\left(K_{\mathrm{ex}}\right)=N \frac{10^{K_{\mathrm{ex}}}}{k_{d}+10^{K_{\mathrm{ex}}}},
$$

first reaches a critical value for the proportion of activated ORPs (typically $1 \%$ ). 
As the shape of this curve is invariant, only its position on the abscissa is shifted as the value of $k_{d}$ changes, the sensitivity for (3.1) can also be defined in terms of the value $K_{\mathrm{ex}}^{50}$ at which $50 \%$ of ORPs are active, $C_{\infty}^{\delta\left(k_{d}\right)}\left(K_{\mathrm{ex}}^{50}\right)=N / 2=\log _{10}\left(k_{d}\right)$ (see Fig. 2). However, if a more flexible curve is used to characterize the inputoutput properties (or an experimentally obtained curve), an intuitive understanding of sensitivity is unclear and several separate characteristics can be used. In Fig. 2, the transfer function for a heterogenous population of ORPs is flatter than the three sigmoid curves to which it is compared. This fact cannot be reflected using a single coordinate of the midpoint, as was done for curve (3.1). Obviously, the part of the curve where the response is regarded as the minimum perceivable, or to be no longer distinguishable from the saturation level may be independent of the midpoint position (cf. Fig. 6).

The coding range of the system can be defined as an interval of concentrations in which the input-output curve gets from $p \%$ of the maxima (detection threshold, $\left.L_{\mathrm{ex}}^{p}\right)$ to $(100-p) \%$ of the maxima (saturation threshold, $L_{\mathrm{ex}}^{100-p}$ ), where selection of $p$ depends on the choice of experimenter $(p=1,5$ and 20 can be used as values for high, moderate, and low levels of resolution). Also, the range over which $L_{\mathrm{ex}}$ is detected is related to the threshold $S$ used in formula (2.18). The situation is different for symmetric curves like those obtained for homogenous population of ORPs (also for some heterogenous cases) and for asymmetric curves. A general condition, as stated in the previous section, for symetricity of the response curve around the midpoint in heterogenous population of ORPs is that the distribution of the dissociation constant is symmetric in the logarithmic scale. In this case the coding range is double the distance between the detection threshold and the midpoint.

It follows from equation (3.1) that for the concentration detector with homogenous population of ORPs holds $L_{\mathrm{ex}}^{p}=\left(p k_{d}\right) /(100-p)$. We can verify that the size of coding range of the concentration detector is $3.99 \log$ units for $p=1$ and 1.91 for $p=10$. This implies that the concentration of activated ligand-ORP complexes increases by almost $10^{4}$ times from detection threshold, at which $1 \%$ of ORPs are in the activated state, to the saturation threshold, at which only $1 \%$ of ORPs are not active. Choosing $p=1$, we calculated $\log _{10}\left(L_{\mathrm{ex}}^{p}\right)$ for binary case illustrated in Fig. 2 to be $\log _{10}\left(L_{\mathrm{ex}}^{1}\right)=-3.69$ and $\log _{10}\left(L_{\mathrm{ex}}^{99}\right)=1.69$ so that the coding range in this case is 6.38 orders of magnitude. The size of the coding range of this binary population of ORPs is more than 50\% larger for this choice of parameters. By taking lower values for $k_{d 1}$ and/or higher values for $k_{d 2}$ we can enlarge the coding range without limit. The limitation to this procedure is that the linear part of the sigmoid transfer function becomes increasingly nonlinear and flat in the middle so that the coding resolution is lost in the middle of the range. The problem of optimal information transfer (linearity of the input-output function) in systems with two different populations of ORPs is solved elsewhere (Getz and Lánský, 2001).

Comparing the above calculated detection threshold $\left(K_{\mathrm{ex}}^{1}=-3.69\right)$ with corresponding values of the components $\left(K_{\mathrm{ex}}^{1}=-4.00\right.$ and $\left.K_{\mathrm{ex}}^{1}=-2.00\right)$, we can see that ORPs with $k_{d}=0.01$ serves mainly as the detectors. Similarly, those with 
$k_{d}=1$ close the coding range and are effective at the saturation threshold. Obviously, if the transfer function is not symmetrical around the midpoint, the coding range can be deduced only from knowledge of both thresholds and is highly dependent on the distributional properties of the ORPs as well as on the relative sizes of the subpopulations. For example, in Fig. 5(a) the parameters used shifts mainly the saturation threshold, in Fig. 5(b) the coding range is enlarged symmetrically, while Fig. 5(c) the detection threshold is moved. More specifically, the detection and saturation thresholds and the coding ranges corresponding to $p=1$ are $(-3.30,1.90)$ in Fig. 5(a), $(-3.69,1,69)$ in Fig. 5(b) and $(-3.90,1.30)$ in Fig. 5(c). The largest coding range is in Fig. 5(b) with differences due primarily to the shift in the central part of the curves. In Fig. 5(a), over the most of the coding range the curve is at low values, the situation in Fig. 5(c) is opposite.

The easiest way to illustrate the effect of heterogeneity and relative independence of the midpoint and detection threshold is to consider the concentration detector with $n$ types of ORPs (i.e., a multinomial distribution of the dissociation constant). In general, the position where curve (2.9) reaches $N / 2$ can be found, but only numerically. In the case when the different ORPs are presented at the same concentrations, i.e., $p_{j}=1 / n$ and when $k_{d j}$ are equally spaced in log scale (it is automatically fulfilled for binomial distribution), value $N / 2$ is reached in the middle $\sqrt[n]{\prod_{j=1}^{n} k_{d j}}$, which is a special case of relation (2.17). On the other hand, if the subpopulations are sufficiently large in comparison with the detection threshold, then the detection is controlled by the subpopulation with fastest reaction and practically does not depend on the remaining types of the ORPs. Analogous conclusion can be derived for saturation threshold.

For the models with arbitrary distribution of dissociation constants, we have shown that inequality (2.13) holds: $C_{\infty}^{x}>C_{\infty}^{\delta\left(k_{d}\right)}$, where $\delta\left(k_{d}\right)$ stands for Dirac distribution at $k_{d}$, where $k_{d}$ is the mean of $K_{d}$, (i.e., $E\left[K_{d}\right]=k_{d}$, see Fig. 2). Thus heterogenous systems always have higher sensitivities than homogenous systems with a dissociation constant equal to the mean of the dissociation constants of the heterogenous population of ORPs irrespective of the definition of sensitivity or the distribution of the dissociation constant. In other words, the detection threshold and the saturation threshold are shifted to lower concentrations in the heterogenous case.

The heterogeneity of ORPs increases the coding range for very obvious reason. For distributions with the same mean and increasing variance, the range of the curve increases. If the distribution is symmetrical and restricted on a bound interval then the largest increase in the coding range is obtained with the binomial distribution concentrated at the end points of this interval (Fig. 4). Thus a normal (truncated to fit on the bounded interval) or uniform distribution contributes less to the coding range comparing than binomial distribution. However, this is true only in the natural scale for the dissociation constant distribution. In log scale the number of bound ORPs for uniform distribution of decadic logarithm of dissociation constant can cover practically any range of stimulus intensity (Fig. 6). 
The previous conclusions concerning the coding properties of membranes with heterogenous populations of ORPs were based on variability of the dissociation constant. The result for fixed dissociation rate and variable association rate, illustrated in Fig. 7, indicates that the coding range is enlarged for heterogenous populations and confined between the transfer curves of homogenous populations in which the mean of the constants and the mean of the distribution are used.

The response curve for symmetric binomial distribution of $K_{d}$ together with twostandard-deviation regions calculated using formula (A1), is illustrated in Fig. 3. The figure suggests the possible variability of the response curve with the largest variability being around the midpoint. Variability of the transfer curve, as illustrated in Fig. 3, provides an explanation of variability observed in experimentally derived transfer curves (Rospars et al., 2000). This is not possible to achieve with homogenous populations of ORPs and therefore the evident statistical fluctuations of the response curves has to be explained in terms of being generated by the other processes down stream from the cell membrane.

The problem of response time is related to the detection problem. We have used a simple example to demonstrate that heterogeneity of ORPs may substantially improve the performance of the response time of a system. Equation (2.18) is highly nonlinear [Fig. 8(a)] and for suitable set of parameters there exists only a very narrow range of stimulation intensities within which the response time gets very close to zero and thus the total reaction time may be practically independent of the response time after stimulation. If there is a subpopulation of ORPs which is sufficiently large and fast in binding, then the response time is the minimum of $\delta_{i}$ given by an equation analogous to equation (2.18) calculated for this subpopulation. Such a subpopulation can be considered just specialized in signaling the presence of the odorant, not its intensity.

\section{Discussion}

At this time, no serious efforts have been made to use ligand-ORP binding theory to assess whether ORNs express one or more ORPs in their membranes. The only direct evidence we have comes from molecular labeling studies (Malnic et al., 1999), and these suggest that in mice each ORN expresses only one kind of ORP. Duchamp-Viret et al. (1999) come to the opposite conclusion using electrophysiological methods to measure the response of individual rat ORNs to stimulation by a suite of structurally dissimilar odorants. Inferring the characteristics of ORP behavior from ORN activity, as in Duchamp-Viret et al. (1999) is difficult for several reasons:

- The spiking rates of individual neurons are hard to relate to the proportion of bound ORPs because neuronal spikes are a series of discrete events while the large numbers of ORPs and odorant molecules make the proportion of 
bound ORPs behave like a continuous variable with dynamics modeled by equation (2.3).

- Even if ORN activity is represented by a continuous variable such as spike rate, several intervening processes (e.g., second messenger cascades, ion channel gating controlling ion flow across ORN membranes, etc.) make the relationship between the two variables (proportion of activated ORPs and ORN spike rate) nonlinear and noisy.

- The process of averaging to obtain the spike rate of an ORN begs the question of time constants involved in ORP binding and other intervening processes, particularly the fact that ORNs may have an initial burst of activity when stimulated, but accommodate by reducing their activity if the stimulus persists [cf. Getz and Akers (1997), Lemon and Getz (1997), also see Lemon and $\operatorname{Getz}(1999,2000)]$.

Despite these difficulties, the range of concentrations over which ORNs respond cannot be greater than the range of concentrations over which ORPs are activated but not saturated (physical laws imply that information on concentration can only be degraded by the intervening processes), although $p$, the lowest proportion of activated ORPs that provides the lowest discernible signal, is not known. Thus we can infer that if an ORN exhibits a graded response over more than 4 orders of magnitude, then a heterogenous ORP population is implied while a response over less than 4 orders of magnitude does not necessarily imply a homogenous ORP population - the ORP population could be heterogenous but the resolution of the ORN low (i.e., $p$ is relatively large).

The best data we are aware of relating ORN response to odorant concentration is in insects. Fujimura et al. (1991) present results on the spike rate of general cockroach olfactory neurons as a function of concentration of a number of odorants including n-alcohols, terpenes, aromatic compounds, acids, and acetates. Unfortunately, most of their plots do not show that full range of response because either the thresholds or saturation points are not apparent. Many of these curves indicate a response range of at least $2-3$ orders of magnitude, suggesting a sensitivity level of at least $p=5 \%$. Cells sensitive to terpineol appear to have response ranges greater than 3, but no ranges of 4 orders of magnitude or greater are indicated: that is, the data show no evidence for ORNs with heterogenous populations of ORPs unless the cells themselves are known to be relatively insensitive (i.e., values for $p$ exceed 5-10\%). Kaissling (as reviewed in 1987) presents data (see his Fig. 29) that indicate pheromonal ORNs in the moth Bombyz mori are sensitive to at least 5-6 orders of magnitude of the odorant bombykol. According to our analysis, this should not be possible unless these pheromone ORNs have at least two subpopulations with ORNs with dissociation constants $k_{d}$ different by 2 or so orders of magnitude.

Thus, Malnic et al.'s (1999) conclusion that the broad spectrum response of individual mammalian ORNs is due to the ORPs themselves being generalists rather than supporting several subpopulations of more specialized ORPs may well hold for generalists insect ORPs. On the other hand, specialized pheromonal ORNs may 
well have heterogenous subpopulations of ORPs to extend the range of pheromone concentrations to which individual cells respond.

Besides providing us with the insight that ORN responses of concentration ranges greater than 4 orders of magnitude strongly suggest that different ORP subpopulations are expressed by individuals ORNs, our model provides the following additional insights (some of which were already known).

- The shape and placement of the proportion of activated ORPs at equilibrium in the concentration detector is determined by value of the ratio $k_{d}=k_{-1} / k_{1}$ but not the individual association and disassociation rates $k_{-1}$ and $k_{1}$ themselves.

- In the context of neuronal response time the chemical reaction rates themselves are important, but for sufficiently strong stimuli the association rate $k_{1}$ alone is the critical determinant.

- In the flux detector, sensitivity is independent of both the association and dissociation rates $k_{1}$ and $k_{-1}$, being dependent rather on odorant influx and degradation rates. This implies that the response time and sensitivity are separated, which is probably not valid for most ORNs. Also the independence of the flux detector on heterogeneity of ORPs limits these types of ORNs from having a their response range enlarged by the expression of several subpopulation of ORPs.

\section{ACKNOWLEDGEMENT}

Petr Lánský thanks the Division of Insect Biology, ESPM, University of California at Berkeley for their hospitality and support during his stay. This work was partly supported by grant MSMT CR (MSM 12 3100004), by Grant Agency of the Czech Academy of Sciences (A7011712) and by NSF Grant IBN 9807938 to WMG.

\section{APpendix A}

For the multinomial distribution of the dissociation constant the variance of the number of activated receptors is

$$
\operatorname{Var}\left(\Gamma_{\infty}^{\mathrm{mn}}\right)=N^{2}\left(\sum_{j=1}^{n}\left(\frac{L_{\mathrm{ex}}}{k_{d j}+L_{\mathrm{ex}}}\right)^{2} p_{j}-\left(\sum_{j=1}^{n} \frac{L_{\mathrm{ex}}}{k_{d j}+L_{\mathrm{ex}}} p_{j}\right)^{2}\right)
$$

If we assume that the dissociation constant is uniformly distributed in $2 \epsilon$ vicinity of $k_{d}$,

$$
f^{u}(x)= \begin{cases}0 & \text { for } 0 \leq x<k_{d}-\epsilon \\ \frac{1}{2 \epsilon} & \text { for } k_{d}-\epsilon \leq x \leq k_{d}+\epsilon \\ 1 & \text { for } k_{d}+\epsilon<x,\end{cases}
$$


and from equation (2.11) we obtain

$$
C_{\infty}^{u}=\frac{N L_{\mathrm{ex}}}{2 \epsilon} \ln \left(\frac{k_{d}+\epsilon+L_{\mathrm{ex}}}{k_{d}-\epsilon+L_{\mathrm{ex}}}\right) .
$$

If in equation (2.14) the range of dissociation constant decreases, then, as $\epsilon \rightarrow 0, C_{\infty}^{u} \rightarrow$ $\frac{N L_{\mathrm{ex}}}{L_{\mathrm{ex}}+k_{d}}$, which is equation (2.10) with $N_{1}=N, k_{d 1}=k_{d}$. The restriction $0<k_{d}-\epsilon$ substantially decreases the difference between response of homogenous and heterogenous population. The variance for uniform distribution of $K_{d}$ is

$$
\operatorname{Var}\left(\Gamma_{\infty}^{u}\right)=N^{2} L_{\mathrm{ex}}^{2}\left(\frac{1}{\left(k_{d}+\epsilon+L_{\mathrm{ex}}\right)\left(k_{d}-\epsilon+L_{\mathrm{ex}}\right)}-\frac{1}{4 \epsilon^{2}} \ln ^{2}\left(\frac{k_{d}+\epsilon+L_{\mathrm{ex}}}{k_{d}-\epsilon+L_{\mathrm{ex}}}\right)\right)
$$

and, as with equation (A1), equation (A3) can be used to create a confidence interval around the transfer function represented by equation (A2).

If we assume a normal distribution of the dissociation constant in equation (2.11) (with suitable variance $\sigma^{2}$ and mean $k_{d}$ to minimize probability of negative values), then we can write

$$
C_{\infty}^{N} \approx N\left(\frac{L_{\mathrm{ex}}}{k_{d}+L_{\mathrm{ex}}}+\frac{\sigma^{2}}{2} \frac{L_{\mathrm{ex}}}{\left(k_{d}+L_{\mathrm{ex}}\right)^{3}}\right)
$$

The condition $k_{d}>0$ implies that the attainable spread of normal distribution is even lower than that of uniform distribution and thus the difference between homogenous and heterogenous populations is even lower than in Fig. 4. The variance of the concentration of bound ORPs for the normal distribution of the dissociation constant can be also calculated.

\section{APPENDiX B}

The mean input-output curve given by (2.10) can be written by using the Taylor expansion of $L_{\mathrm{ex}} /\left(x+L_{\mathrm{ex}}\right)$ in point $\mu$ as

$$
E\left(C_{\infty}\right)=N \int_{0}^{\infty} \sum_{n=0}^{\infty} \frac{(-1)^{n} L_{\mathrm{ex}}}{\left(\mu+L_{\mathrm{ex}}\right)^{n+1}} \frac{(x-\mu)^{n}}{n !} d F(x) .
$$

If all the moments of random variable characterized by the cumulative distribution function $F$ exist, then the sum and integral can be replaced. If we select $\mu$ to be the mean of $K_{d}$ then the first term $(n=0)$ in $(\mathrm{B} 1)$ is the 'mean' term

$$
N \frac{L_{\mathrm{ex}}}{\mu+L_{\mathrm{ex}}}
$$

For cumulative distribution functions of symmetrical distributions hold $F(\mu-x)=1-$ $F(\mu+x)$, [if density exists, then $f(\mu-x)=f(\mu+x)$ ]. Thus the terms with odd $n$ are zero due to the symetricity of the distribution and terms for which $n$ is even are positive, so (2.13) is proved. It follows from the shape of $\frac{L_{\mathrm{ex}}}{\left(\mu+L_{\mathrm{ex}}\right)^{n+1}}$ that the shift of the curves is mainly for low level of $L_{\mathrm{ex}}$ and increasing with the variance of the distribution $F$. 


\section{Appendix C}

Let us consider the case where we assume $K_{1}$ has a uniform distribution over the interval $\left[k_{1}-\epsilon, k_{1}+\epsilon\right]$

$$
g^{u}(x)= \begin{cases}0 & \text { for } 0 \leq x \leq k_{1}-\epsilon \\ \frac{1}{2 \epsilon} & \text { for } k_{1}-\epsilon \leq x \leq k_{1}+\epsilon \\ 1 & \text { for } k_{1}+\epsilon<x,\end{cases}
$$

and $k_{-1}$ is constant. Then the probability density function of $K_{d}$ has the form

$$
f(x)=\frac{k_{-1}}{2 x^{2} \epsilon}, \quad x \in\left(\frac{k_{-1}}{k_{1}+\epsilon}, \frac{k_{-1}}{k_{1}-\epsilon}\right) .
$$

Now, it follows that $K_{d}$ is no longer centered around $k_{-1} / k_{1}$, as it was in density used in Appendix A. Further, the mean of distribution (C1) is $\left[k_{-1} \ln \left\{\left(k_{1}+\epsilon\right) /\left(k_{1}-\varepsilon\right)\right\}\right] /(2 \epsilon)$ which tends to $k_{-1} / k_{1}$ as $\epsilon \rightarrow 0$. Again, we see that $f(x)$ is positively skewed with the majority of ORPs having a realized values of $K_{d}$ higher than $k_{-1} / k_{1}$. From equation (2.11) we obtain

$$
E\left(C_{\infty}^{1 / u}\right)=N\left(1+\frac{k_{-1}}{2 L_{\mathrm{ex}} \epsilon} \ln \left(\frac{k_{-1}+\left(k_{1}-\epsilon\right) L_{\mathrm{ex}}}{k_{-1}+\left(k_{1}+\epsilon\right) L_{\mathrm{ex}}}\right)\right) .
$$

\section{REFERENCES}

Beidler, B. W. (1962). Taste receptor stimulation. Prog. Biophys. Chem. 12, 107-151.

Buck, L. B. (1996). Information coding in the vertebrate olfactory system. Annu. Rev. Neurosci. 19, 517-544.

Buck, L. B. and R. Axel (1991). A novel multigene family may encode odorant receptorsa molecular basis for odor recognition. Cell 65, 175-187.

Duchamp-Viret, P., M. A. Chaput and A. Duchamp (1999). Odor response properties of rat olfactory receptor neurons. Science 284, 2171-2174.

Ennis, D. M. (1991). Molecular mixture models based on competitive and noncompetitive agonism. Chem. Senses 16, 1-17.

Fujimura, K., F. Yokohari and H. Tateda (1991). Classification of antenal olfactory receptors of the cockroach. Periplaneta americana. Zoological Sci. 8, 243-255.

Getz, W. M. (1999). A kinetic model of the transient phase in the response of the olfactory receptor neuron. Chem. Senses 24, 497-508.

Getz, W. M. and R. P. Akers (1995). Partitioning non-linearities in the response of olfactory neurons to binary odors. BioSystems 34, 27-40.

Getz, W. M. and R. P. Akers (1997). Coding properties of peak and average response rates in American cockroach olfactory cells. BioSystems 40, 55-63.

Getz, W. M. and P. Lánský (2001). Ligand concentration coding and optimal MichaelisMenten parameters in multivalent and heterogenous receptor membrane. Chem. Senses 26, 95-104.

Kaissling, K.-E. (1986). Chemo-electrical transduction in insect olfactory receptors. Ann. Rev. Neurosci. 9, 121-145.

Kaissling, K.-E. (1987). R.H. Wright Lectures on Insect Olfaction, Burnaby: Simon Fraser University.

Kaissling, K.-E. (1998). Flux detectors vs. concentration detectors: two types of chemoreceptors. Chem. Senses 23, 99-111. 
Lancet, D., R. Gross-Isseroff, T. Margalit, E. Seidemann and N. Ben-Arie (1993a). Olfaction: From signal transduction and termination to human genome mapping. Chem. Senses 18, 217-225.

Lancet, D., E. Sadovsky and E. Seidemann (1993b). Probability model for molecular recognition in biological receptor repertoires-significance to the olfactory system. PNAS 90, 3715-3719.

Lánský, P., V. Krivan and J.-P. Rospars (2001). Ligand interaction with receptors under periodic stimulation: A modeling study with application to concentration chemoreceptors. Eur. Biophys. J. (in press).

Lánský, P. and J.-P. Rospars (1993). Coding of odor intensity. BioSystems 31, 15-38.

Lánský, P. and J.-P. Rospars (1995). Mathematical approach to transduction processes in olfactory receptor neurons. J. Jap. Soc. Instrument Control Engn. 34, 800-804.

Lánský, P. and J.-P. Rospars (1998). Odorant concentration and receptor potential in olfactory sensory neurons. BioSystems 48, 131-138.

Lemon, W. C. and W. M. Getz (1997). Temporal resolution of general odor pulses by olfactory sensory neurons in American cockroaches. J. Exp. Biol. 200, 1809-1819.

Lemon, W. C. and W. M. Getz (1999). Olfactory coding in insects. Ann. Ent. Soc. Am. 92, 861-872.

Lemon, W. C. and W. M. Getz (2000). Rate code input produces temporal code output from cockroach antennal lobes. BioSystems 58, 151-158.

Malaka, R., T. Ragg and M. Hammer (1995). Kinetic models of odor transduction implemented as artificial neural networks. Biol. Cybern. 73, 195-207.

Malnic, B., J. Hirono, T. Sato and L. B. Buck (1999). Combinatorial receptor codes for odores. Cell 96, 713-723.

Masson, C. and H. Mustaparta (1990). Chemical information processing in olfactory system of insects. Physiol. Rev. 70, 199-245.

Mombaerts, P. (1999). Seven-transmembrane proteins as odorant and chemosensory receptors. Science 286, 707-711.

Rospars, J.-P., V. Krivan and P. Lánský (2000). Perireceptor and receptor events in olfaction. Comparison of concentration and flux detectors: A modeling study. Chem. Senses 25, 293-311.

Rospars, J.-P., P. Lánský, H. C. Tuckwell and A. Vermeulen (1996). Coding of odor intensity in a steady-state deterministic model of an olfactory receptor neuron. J. Comput. Neurosci. 3, 51-72.

Smith, B. H. and W. M. Getz (1994). Nonpheromonal olfactory processing in insects. Annu. Rev. Entomol. 39, 351-375. 